\title{
Use of infrared thermography to estimate brown fat activation after a cooling protocol in patients with severe obesity that underwent bariatric surgery
}

\section{Short title: Brown adipose tissue after bariatric surgery}

Irene Piquer-Garcia ${ }^{1}$, Rubén Cereijo ${ }^{2,3}$, Juan Corral-Pérez ${ }^{4,5}$, Silvia Pellitero ${ }^{1,6}$, Eva Martínez ${ }^{1}$, Siri

D. Taxerås ${ }^{1}$, Jordi Tarascó ${ }^{1}$, Pau Moreno ${ }^{1}$, José Balibrea ${ }^{7}$, Manel Puig-Domingo ${ }^{1,6}$, Dolors Serra $^{3,8}$, Laura Herrero ${ }^{3,8}$, David Jiménez-Pavón ${ }^{4,5}$, Carles Lerin ${ }^{9}$, Francesc Villarroya ${ }^{2,3}$, David Sánchez-Infantes ${ }^{1,3^{*}}$

${ }^{1}$ Germans Trias i Pujol Research Institute, Barcelona, Spain

${ }^{2}$ Department of Biochemistry and Molecular Biomedicine, and Institute of Biomedicine, University of Barcelona, Barcelona, Spain.

${ }^{3}$ Centro de Investigación Biomédica de Fisiopatología de la Obesidad y la Nutrición (CIBEROBN), Instituto de Salud Carlos III, E-28029 Madrid, Spain.

${ }^{4}$ MOVE-IT Research Group and Department of Physical Education, Faculty of Education Sciences University of Cádiz, Cádiz, Spain.

${ }^{5}$ Institute of Research and Innovation in Biomedical Sciences of the Province of Cádiz (INiBICA), University of Cádiz, Cádiz, Spain.

${ }^{6}$ Centro de Investigación Biomédica en Fisiopatología de la Diabetes y enfermedades metabólicas (CIBERDEM), ISCIII, Madrid, Spain.

${ }^{7}$ Metabolic and Bariatric Surgery Unit, EAC-BS Center of Excellence, Vall d'Hebron University Hospital, Barcelona, Spain.

${ }^{8}$ Department of Biochemistry and Physiology, School of Pharmacy, Institut de Biomedicina de la Universitat de Barcelona (IBUB), Universitat de Barcelona, E-08028 Barcelona, Spain

${ }^{9}$ Endocrinology department, Institut de Recerca Sant Joan de Déu, 08950 Barcelona, Spain.

${ }^{*}$ Correspondence:

David Sánchez-Infantes, PhD dsanchez@igtp.cat

Germans Trias i Pujol Research Institute (IGTP)

Campus Can Ruti. Carretera de Can Ruti, Camí de les Escoles s/n

08916 Badalona, Barcelona, Spain Tel: (+34) 935572832 Fax: (+34) 934978654

\section{Acknowledgement}

Samples from patients included in this study were provided by the IGTP HUGTP Biobank integrated in the Spanish National Biobanks Network of "Instituto de Salud Carlos III" 
(PT13/0010/0009) and they were processed following standard operating procedures with the appropriate approval of the Ethical and Scientific Committees.

\section{Funding agencies:}

DS-I is an Investigator of the Miguel Servet Fund from Carlos III National Institute of Health, Spain (CP15/00106). This study was supported by Instituto de Salud Carlos III, and by the Fondo Europeo de Desarrollo Regional (FEDER), Madrid, Spain (FIS17/01455) and by K-sted 651010-Project number 70084900-analyse number M50676 from Norwegian University of Science and Technology (NTNU). DJP was supported by a grant from the Spanish Ministry of Science and Innovation - MINECO (RYC-2014-16938). This study was supported by the Ministry of Spain (MINECO) (Grant SAF2017-83813-C3-1-R to DS and LH cofunded by the European Regional Development Fund [ERDF]), the Centro de Investigación Biomédica en Red de Fisiopatología de la Obesidad y la Nutrición (CIBEROBN) (Grant CB06/03/0001 to DS), and the Fundació La Marató de TV3 (Grant 87/C/2016 to DS).

\section{COI statement:}

Irene Piquer-Garcia has nothing to disclose.

Rubén Cereijo has nothing to disclose.

Juan Corral-Pérez has nothing to disclose.

Silvia Pellitero has nothing to disclose.

Eva Martínez has nothing to disclose.

Siri D. Taxerås has nothing to disclose.

Jordi Tarascó has nothing to disclose.

Pau Moreno has nothing to disclose.

José Balibrea has nothing to disclose.

Manel Puig-Domingo has nothing to disclose.

Dolors Serra has nothing to disclose.

Laura Herrero has nothing to disclose.

David Jiménez-Pavón has nothing to disclose.

Carles Lerin has nothing to disclose.

Francesc Villarroya reports grants from Ministerio de Ciencia, Innovació y Universidades, outside the submitted work.

David Sánchez-Infantes has nothing to disclose. 


\title{
Use of infrared thermography to estimate brown fat activation after a cooling protocol in patients with severe obesity that underwent bariatric surgery
}

\begin{abstract}

\section{Introduction}

Obesity has reached epidemic proportions worldwide, and its prevalence has doubled in the last 30 years. In contrast to the energy-storing role of white adipose tissue (WAT), brown adipose tissue (BAT) acts as the main site of non-shivering thermogenesis in mammals, and has been reported to play a major role in protection against obesity and associated metabolic alterations in rodents. Infrared thermography (IRT) has been proposed as a novel non-invasive, safe, inexpensive, and quick method to estimate BAT thermogenic activation in humans. The aim of this study is to determine the thermogenic activation of BAT after a cooling protocol using IRT in patients with severe obesity in response to two different types of bariatric surgery (BS).
\end{abstract}

\section{Methods}

Supraclavicular BAT thermogenic activation was evaluated using IRT in a cohort of 31 patients ( $50 \pm 10$ years-old, $\mathrm{BMI}=44.5 \pm 7.8$ ) at baseline and 6 months after BS. Clinical parameters were determined at different time points.

\section{Results}

BAT thermogenic activation by IRT was increased at 6 months after laparoscopy sleeve gastrectomy (LSG), while patients undergoing to a roux-en-Y gastric bypass (RYGB) did not change their thermogenic response using the same cooling protocol.

\section{Conclusions}

Our study reports a differential effect of LSG technique compared to RYGB on BAT activation, suggesting that the mechanisms involved in weight loss after surgery might differ between the two techniques. 


\section{Introduction/Purpose}

Increased body mass is associated with numerous metabolic diseases, including type 2 diabetes (T2D). Thus, it is not surprising to find out that, concomitantly with the dramatic increase in obesity, T2D has become the most common metabolic disorder, being recognized as one of the deadliest non-communicable diseases worldwide. Nowadays, it has been estimated that approximately 350 million people has diabetes, representing almost $10 \%$ of the world population [1]. White adipose tissue (WAT) is involved in the regulation of energy balance and glucose homeostasis, owing to its function as a lipid-storing and endocrine organ. Numerous studies have demonstrated that abnormal WAT function is linked to obesity, whole body insulin resistance and T2D [2]. However, few studies have comprehensively addressed the effect of obesity and weight loss in the thermogenic capacity.

In contrast to the energy-storing role of WAT, brown adipose tissue (BAT) acts as the main site of non-shivering thermogenesis in mammals due to the presence of uncoupling protein-1 (UCP1) in mitochondria of brown adipocytes, which uncouples mitochondrial oxidative processes and generates heat [3]. BAT plays a major role in protection against obesity and associated metabolic alterations in rodents due to its draining of glucose and lipids from circulation to sustain thermogenesis $[4,5]$. Moreover, sustained thermogenic activation leads also to the so-called 'browning' of adipose tissue: the appearance of beige (brown adipocytelike) adipocytes in anatomical sites corresponding to WAT depots [6, 7]. Chronic exercise, cold exposure and chronic $\beta 3$-adrenergic stimulation have been shown to promote browning of WAT in experimental models [8]. A higher capacity of browning has been directly associated with protection against experimental obesity and improved glucose tolerance in mice [9]. Moreover, BAT has been also reported to secrete endocrine regulatory factors which contribute, in addition to the intrinsic energy expending properties of BAT, to the healthy effects of active BAT on systemic metabolism $[10,11]$.

Laparoscopy sleeve gastrectomy (LSG) and Roux-en-Y gastric bypass (RYGB) are two types of bariatric surgery (BS), the most effective therapy to avert obesity and T2D currently available [12-15]. One of the consequences of BS is activation of BAT and browning of WAT, which is hypothesized to contribute to the increased energy expenditure, weight loss, and overall improvement in systemic glucose and lipid metabolism after BS [16, 17]. In 2009, several studies confirmed that active BAT is present in adult humans, and that, accordingly, BAT activity is reduced in patients with obesity [18-21]. One of the techniques currently used to quantify BAT is positron emission tomography with 2-deoxy-2-[fluorine-18] fluoro-D-glucose integrated with computed tomography (18F-FDG-PET-CT). This technique is considered the "gold-standard" to measure BAT/beige activity and metabolism [22], it is an expensive, time-consuming and 
relatively invasive technique [23]. For the moment, the lack of non-invasive and low-cost methods to measure BAT activation before and after BS in patients makes it difficult to implement this parameter in the clinical practice for a better evaluation of the patients.

In the last years, infrared thermography (IRT) has been proposed as a novel noninvasive, safe, cheap and quick method to estimate BAT thermogenic activation in humans [24]. IRT uses the heat-emitting properties of BAT and the relatively superficial position of the supraclavicular BAT depot. A rise in supraclavicular temperatures after cold exposure has been demonstrated using this methodological approach, and several studies correlated IRT data obtained in supraclavicular skin surface with BAT activity as determined using PET scans [25-29].

In this study, we determined for the first time the thermogenic activation of BAT after a hand-cold protocol using IRT in patients with severe obesity and the role of two different types of BS techniques.

\section{Materials and Methods}

\section{Subject cohort and surgical interventions}

A cohort of 31 patients ( 19 females $/ 12$ males, aged= $50 \pm 10$ years-old, $B M I=44.5 \pm 7.8$ ) with severe obesity were included in this study, and were stratified into two groups according to the type of BS they underwent: 1$)$ LSG ( $n=15)$, in which the stomach is transected vertically from $5 \mathrm{~cm}$ proximal to the pylorous up to the His' angle, using a $36 \mathrm{Fr}$ bougie as a calibrator. Hence, more than $70 \%$ of patient's stomach (mainly fundus and body) volume is removed without modifying the rest of gastrointestinal tract (GIT) [30]; 2) RYGB ( $n=16)$, where the surgeons tailor a 40-60 $\mathrm{mL}$ gastric pouch that is anastomosed to the previously divided jejunum distal to the ligament of Treitz. Reconstruction of the GIT was completed in a " $Y$ " configuration with a distal jejuno-jejunostomy [31]. All patients were evaluated by the same endocrinology specialist according to criteria formulated in Spanish Position Statement between Obesity, Endocrinology, Diabetes and Surgery Societies [32]. IRT, demographic and clinical data, including age, diabetes, and hypertension were recorded for all participants at baseline and 6 months after BS.

\section{Study Visits and Protocols}

For IRT acquisition, participants went to a specific room where the body area object of evaluation was uncovered and sat-down during $5 \mathrm{~min}$ in a thermoneutral ambient for acclimatization $(24.3 \pm 1.6 \circ \mathrm{C})$. Women were asked to move the straps of their sports bra aside, and any long-haired participants were asked to tie their hair up to expose the supraclavicular area when necessary. 
For the cold protocol, 3 thermal images were taken for each patient using a FLIR T420

infrared camera (FLIR T420 Systems AB, Sweden), with a thermal sensitivity of $0.05 \stackrel{\circ}{\circ}$ and resolution set at $320 \times 240$ pixels. The first image was taken to an aluminum foil phantom ( 1 meter away) to obtain a measurement of the reflected temperature for each set of images; in this moment, ambient temperature and relative humidity were registered for each set as well. For the second thermal image, the participants remained seated in an upright position, with their arms relaxed on both sides of their legs. After a calculation of optimal distances, the camera was placed 1 meter from the midpoint of the chair for these images. For the third image, patients were asked to put their left hand in cold water (17으) during 5 minutes to stimulate BAT activation, after which the thermographic picture was taken.

\section{Analysis of IRT}

Thermal data were extracted from IRT pictures by using a region of interest (ROI)-based approach. The ROls were manually drawn in the images on the supraclavicular and sternum region using the FLIR ResearchIR Max software version 4.40.6.24 for windows (FLIR Systems Inc., North Billerica, MA, USA). All analyses were adjusted by atmospheric temperature, distance to the participant and relative humidity, which, as previously stated, were recorded at the beginning of each IRT session. Moreover, the reflected temperature was obtained by placing a rounded ROI on the aluminum foil phantom of the first thermal image and retaining the mean value $(\stackrel{\circ}{ }$ ) for adjustments. For all thermographic images emissivity was set at 0.98 (human skin). Minimum, maximum and mean values of each $\mathrm{ROI}$ were retained as variables. The temperature in the supraclavicular region was normalized by sternum region temperature for each participant at all time points before and after BS.

We calculated the delta 'Supraclavicular T - Sternum T in thermal image number 1 (0 min) and in thermal image number 2 ( 5 min after cold stimulation), obtaining a value ' $\Delta T^{0 \prime}$ for the thermal image 1 and a value ' $\Delta \mathrm{T}^{5 \prime}$ for the thermal image 2 :

$$
\begin{aligned}
& \Delta T_{(\text {scr-str })}^{0}=T_{\text {supraclavicular area }}^{0}-T_{\text {sternum area }}^{0} \\
& \Delta T_{(\text {scr-str })}^{5}=T_{\text {supraclavicular area }}^{5}-T_{\text {sternum area }}^{5}
\end{aligned}
$$

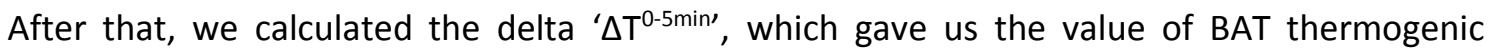
activation capacity elicited by cold stimulus. This is key readout of our study:

$$
\Delta T^{0-5 \min }=\Delta T_{(s c r-s t r)}^{0}-\Delta T_{(s c r-s t r)}^{5}
$$

We repeated this same sequence 6 months after bariatric surgery, and we compared the values of the capacity of BAT thermogenic activation by cold stimulus 6 months after surgery, taking into account the different type of surgery. 


\section{Serum samples}

Serum samples from the study participants were collected after a $12 \mathrm{~h}$ fasting period at baseline and 6 months after BS. All samples were stored at $-80^{\circ} \mathrm{C}$ in the Biobanc of the Health Sciences Research Institute Germans Trias i Pujol Foundation. The Institutional Ethics Committee, in accordance with the Declaration of Helsinki, approved the study (PI16-025). All participants gave their written informed consent before the IRT and the collection of clinical data and samples.

\section{Human serological analysis}

Fasted glucose and insulin levels, glycated haemoglobin, lipid profile (total cholesterol, HDL and LDL cholesterol, and triglycerides), urea, creatinine, and c-reactive protein were measured in the certified core clinical laboratory at the hospital. Body mass index, waist circumference and blood pressure were measured by the endocrinologists and dieticians in charge of the patients. The Homeostatic model Assessment-insulin resistance (HOMA-IR) was calculated through the following formula:

$$
\text { HOMA }-I R=\frac{\left[\text { Glucose } \frac{m g}{d L}\right] *\left[\operatorname{Insulin} \frac{\mathrm{m} . \mathrm{u} . \mathrm{int}}{d L}\right]}{405}
$$

\section{Statistical analysis}

Data are presented as mean \pm sem. Statistical tests were performed with GraphPad Prism 6.0. Normality of datasets was assessed using the Kolmogorov-Smirnoff test. Wilcoxon matched pairs test was used to compare delta ' $\Delta T^{0-5 \mathrm{~min}}$ ' before and after bariatric surgery. Grubbs test was used to remove outliers prior to statistical analyses. $P<0.05$ was considered as the threshold of statistical significance in all analyses.

\section{Results}

BAT thermogenic capacity of patients with severe obesity increases after BS. Evidence of higher effects of the LSG versus RYGB type of BS on the induction of BAT activation.

Clinical data from patients with obesity are shown in table 1. Patients were distributed according to the type of surgery. As expected, both LSG and RYGB led to weight loss and improved metabolic profile, including glucose and triglycerides levels. The group of individuals operated by LSG displayed significant lower levels of glucose and glycated hemoglobin before the surgery compared to the RYGB group. IRT images from all participants were taken at baseline (before surgery) and 6 months after the surgery. 
We performed a pilot study in order to select the correct time of cold stimulus, and the cold water. We detected a marked variability at very short times between the different patients (Supplementary Figure S1), and considering our observations and the previous literature, we chose to perform 5 minutes of cold stimulus.

Figure 1 shows a representative example of IRT images of one participant from each type of surgery. This figure shows the lack of thermogenic activation by cold exposure before surgery in both patients. At 6 months after bariatric surgery, cold exposure activated supraclavicular BAT in the patient from the LSG group but not in the patient from the RYGB group. The general data from the whole cohort of patients are shown in Figure 2, where we observe a significant increase of the thermogenic activity 6 months after LSG but not RYGB. There were no significant differences between females and males before and after bariatric surgery.

\section{Conclusions}

In this study, we demonstrate for the first-time usage of IRT as a novel non-invasive technique to estimate BAT activation after hand-cold exposure in patients with severe obesity undergoing BS. Due to IRT being a non-invasive method, a longitudinal approach could be used to estimate BAT activity at several time points after BS in patients with obesity.

Patients undergoing BS are currently evaluated before surgery in different aspects including endocrine-metabolic status and psychological traits. However, it is still challenging to predict the success of surgery because of the high variability in the extent of weight loss after surgery. Moreover, it is known that BAT thermogenic activation inversely correlates with BMI [18]. According with that, we have observed that at 6 months after BS, BAT thermogenic capacity increases. Therefore, we propose that this parameter, obtained through a non-invasive method such as IRT, is worthy to be considered among the number of data that are taken into account to evaluate the physiological and metabolic status of patients candidates to BS.

Interestingly, we found differences in the extent of BAT activation depending of the type of surgery, being patients undergoing LSG but not RYGB the ones who actually improved BAT activation capacity after BS. It is important to point out that RYGB has demonstrated better longterm results in patients with diabetes [33], and due to this fact, it is accepted in the clinical practice that this type of surgery must be performed in patients with severe obesity and impaired glucose homeostasis. In our study, differences in glucose levels and glycated hemoglobin were found between LSG and RYGB groups at baseline. Moreover, the LSG group showed normal glucose levels despite of having the same degree of obesity in terms of BMI 
compared to RYGB group. Considering that BAT has demonstrated its positive role in energy speculate that the BAT of patients with normoglycemia would maintain a better function than patients with pre-diabetes or diabetes in terms of endocrine activity. It could explain, at least in part, the improvement in insulin sensitivity and the increase in the capacity to activate BAT after cold exposure observed in LSG group compared to RYGB, which might be contributing to the success of BS regarding weight loss. Other researchers have described BAT activation measured by 18F-FDG-PET-CT after a RYGB, but interestingly, a lack of hypothalamic activity was reported in these patients with obesity before and after the RYGB [17]. Since hypothalamic signaling to BAT is the main neural circuit involved in thermogenic activation of this tissue [34], a potential explanation is that our protocol to expose patients to cold stress ( 5 minutes with their hand in cold water) might not be enough to activate BAT thermogenesis in the RYGB group compared to LSG group due to this impairment of sympathetic signaling in the former set of patients. Other mechanisms of action (e.g. specific changes in enterokine secretion, adipokine release or microbiota changes) could account for the systemic effects of RYGB without involving central nervous BAT activation. Further studies should be developed to decipher the potential different effect of LSG and RYGB in the capacity to activate BAT thermogenesis.

Despite the advantages described above, IRT-based estimation of BAT activity is not exempt of limitations. Some researchers have pointed out some flaws in the quantification of BAT activity by IRT in subjects with obesity, suggesting that the changes in the layer of subcutaneous fat insulation could be a confounding factor to measure BAT temperature [35]. Although we assume this potential limitation in our study, it is important to remark that we did not use basal temperature at the supraclavicular area as index of BAT activity but the individual capacity to increase supraclavicular temperature before and after a single bout of cold stress in every individual. As the fat layer is the same before and after the $5 \mathrm{~min}$ cold-stimulus exposure in each individual patient, changes in fat layer width are not expected to significantly influence our estimation. PET-scan-based measurement of BAT activity may have less potential limitations in this regard. However, using PET-scan techniques rises as well a number of concerns (e.g., specific type of labeled metabolite used for the assay, stress associated with the scanning procedures, etc.) and are obviously not feasible for dynamic repeated measuring in the same individual as performed in our study, which can otherwise be assessed by IRT. Moreover, the lack of randomization in the type of surgery and no inclusion of normal weight individuals to compare at the same time their thermogenic activation by IRT are also limitations of the study and might be a bias in the different response to RYGB and LSG. 
In conclusion, we report for the first time that IRT-based estimation of BAT thermogenic activity may be useful for a follow-up of patients after BS in a convenient, non-invasive manner. Thus, we propose that quantification of active BAT by IRT after a cold protocol may be useful in clinical practice as an additional measurement to evaluate the metabolic status and the potential success of BS in patients with obesity. Further studies including a meta-analysis to check potential biological and clinical associations would be necessary to establish its precise contribution to the clinical practice.

\section{Conflict of Interest}

The authors declared no conflict of interest. 


\section{References}

1) WHO. Obesity and overweight. World Health Organization; 2017.

2) Apostolopoulos V, de Courten MP, Stojanovska L, Blatch GL, Tangalakis K, de Courten B. The complex immunological and inflammatory network of adipose tissue in obesity. Mol Nutr Food Res. 2016;60(1):43-57. doi: 10.1002/mnfr.201500272

3) Villarroya J, Cereijo R, Villarroya F. An endocrine role for brown adipose tissue? Am J Physiol Endocrinol Metab. 2013;305(5):E567-572. doi: 10.1152/ajpendo.00250.2013

4) Bartelt A, Bruns OT, Reimer R, Hohenberg H, Ittrich H, Peldschus K, Kaul MG, Tromsdorf UI, Weller H, Waurisch C, Eychmuller A, Gordts PL, Rinninger F, Bruegelmann K, Freund $B$, Nielsen $\mathrm{P}$, Merkel $\mathrm{M}$, Heeren J. Brown adipose tissue activity controls triglyceride clearance. Nat Med. 2011;17(2):200-205 doi: 10.1038/nm.2297.

5) Peirce V, Vidal-Puig A. Regulation of glucose homoeostasis by brown adipose tissue. Lancet Diabetes Endocrinol. 2013;1(4):353-360. doi: 10.1016/S2213-8587(13)70055-X

6) Petrovic N, Walden TB, Shabalina IG, Timmons JA, Cannon B, Nedergaard J. Chronic peroxisome proliferator-activated receptor gamma (PPARgamma) activation of epididymally derived white adipocyte cultures reveals a population of thermogenically competent, UCP1-containing adipocytes molecularly distinct from classic brown adipocytes. J Biol Chem. 2010;285(10):7153-7164. doi: 10.1074/jbc.M109.053942

7) Wu J, Bostrom P, Sparks LM, Ye L, Choi JH, Giang AH, Khandekar M, Virtanen KA, Nuutila P, Schaart G, Huang $K$, Tu $H$, van Marken Lichtenbelt WD, Hoeks J, Enerback S, Schrauwen P, Spiegelman BM. Beige adipocytes are a distinct type of thermogenic fat cell in mouse and human. Cell. 2012;150(2):366-376. doi: 10.1016/j.cell.2012.05.016

8) Wankhade UD, Shen M, Yadav H, Thakali KM. Novel Browning Agents, Mechanisms, and Therapeutic Potentials of Brown Adipose Tissue. Biomed Res Int. 2016;2016:2365609. doi: $10.1155 / 2016 / 2365609$

9) Seale P, Conroe HM, Estall J, Kajimura S, Frontini A, Ishibashi J, Cohen P, Cinti S, Spiegelman BM. Prdm16 determines the thermogenic program of subcutaneous white adipose tissue in mice. J Clin Invest. 2011;121(1):96-105. doi: 10.1172/JCI44271

10) Villarroya F, Cereijo R, Villarroya J, Giralt M. Brown adipose tissue as a secretory organ. Nat Rev Endocrinol. 2017;13(1):26-35. doi: 10.1038/nrendo.2016.136

11) Cereijo R, Sánchez-Infantes D. Brown adipose tissue and browning: More than just a heating device. Endocrinol Diabetes. 2017 64(4):185-187. doi: 10.1016/j.endinu.2017.03.001

12) Aron-Wisnewsky J, Tordjman J, Poitou C, Darakhshan F, Hugol D, Basdevant A, Aissat A, Guerre-Millo M, Clement K. Human adipose tissue macrophages: $\mathrm{m} 1$ and $\mathrm{m} 2$ cell surface 
markers in subcutaneous and omental depots and after weight loss. J Clin Endocrinol Metab. 2009;94(11):4619-4623. doi: 10.1210/jc.2009-0925

13) Pontiroli AE, Morabito A. Long-term prevention of mortality in morbid obesity through bariatric surgery: a systematic review and meta-analysis of trials performed with gastric banding and gastric bypass. Ann Surg. 2011;253:484-487. doi: $10.1097 /$ SLA.0b013e31820d98cb

14) Poitou C, Perret C, Mathieu F, Truong V, Blum Y, Durand H, Alili R, Chelghoum N, Pelloux V, Aron-Wisnewsky J, Torcivia A, Bouillot JL, Parks BW, Ninio E, Clement K, Tiret L. Bariatric Surgery Induces Disruption in Inflammatory Signaling Pathways Mediated by Immune Cells in Adipose Tissue: A RNA-Seq Study. PloS one. 2015;10(5):e0125718. doi: 10.1371/journal.pone.0125718

15) Cummings DE, Cohen RV. Bariatric/Metabolic Surgery to Treat Type 2 Diabetes in Patients With a BMI <35 kg/m2. Diabetes Care. 2016;39(6):924-933. doi: 10.2337/dc160350

16) Vijgen GH, Bouvy ND, Teule GJ, Brans B, Hoeks J, Schrauwen P, van Marken Lichtenbelt WD. Increase in brown adipose tissue activity after weight loss in morbidly obese subjects. J Clin Endocrinol Metab. 2012;97(7):E1229-1233. doi: 10.1210/jc.2012-1289

17) Rachid B, van de Sande-Lee S, Rodovalho S, Folli F, Beltramini GC, Morari J, Amorim BJ, Pedro T, Ramalho AF, Bombassaro B, Tincani AJ, Chaim E, Pareja JC, Geloneze B, Ramos CD, Cendes F, Saad MJ, Velloso LA. Distinct regulation of hypothalamic and brown/beige adipose tissue activities in human obesity. Int J Obes. 2015;39(10):1515-1522. doi: 10.1038/ijo.2015.94

18) Cypess AM, Lehman S, Williams G, Tal I, Rodman D, Goldfine AB, Kuo FC, Palmer EL, Tseng YH, Doria A, Kolodny GM, Kahn CR. Identification and importance of brown adipose tissue in adult humans. N Engl J Med. 2009;360(15):1509-1517. doi: 10.1056/NEJMoa0810780

19) Saito M, Okamatsu-Ogura $Y$, Matsushita M, Watanabe K, Yoneshiro T, Nio-Kobayashi J, Iwanaga T, Miyagawa M, Kameya T, Nakada K, Kawai Y, Tsujisaki M. High incidence of metabolically active brown adipose tissue in healthy adult humans: effects of cold exposure and adiposity. Diabetes. 2009;58(7):1526-1531. doi: 10.2337/db09-0530

20) van Marken Lichtenbelt WD, Vanhommerig JW, Smulders NM, Drossaerts JM, Kemerink GJ, Bouvy ND, Schrauwen P, Teule GJ. Cold-activated brown adipose tissue in healthy men. N Engl J Med. 2009;360(15):1500-1508. doi: 10.1056/NEJMoa0808718 
21) Virtanen KA, Lidell ME, Orava J, Heglind $M$, Westergren $R$, Niemi $T$, Taittonen $M$, Laine J, Savisto NJ, Enerback S, Nuutila P. Functional brown adipose tissue in healthy adults. N Engl J Med. 2009;360(15):1518-1525. doi: 10.1056/NEJMoa0808949

22) Dadson P, Hannukainen JC, Din MU, Lahesmaa M, Kalliokoski KK, lozzo P, Pihlajamäki J, Karlsson HK, Parkkola R, Salminen P, Virtanen KA, Nuutila P. Brown adipose tissue lipid metabolism in morbid obesity: Effect of bariatric surgery-induced weight loss. Diabetes Obes Metab. 2018;20(5):1280-1288. doi: 10.1111/dom.13233

23) Chen KY, Cypess AM, Laughlin MR, Haft CR, Hu HH, Bredella MA, Enerback S, Kinahan PE, Lichtenbelt W, Lin FI, Sunderland JJ, Virtanen KA, Wahl RL. Brown Adipose Reporting Criteria in Imaging STudies (BARCIST 1.0): Recommendations for Standardized FDGPET/CT Experiments in Humans. Cell Metab. 2016;24(2):210-222. doi: 10.1016/j.cmet.2016.07.014

24) Law J, Chalmers J, Morris DE, Robinson L, Budge H, Symonds ME. The use of infrared thermography in the measurement and characterization of brown adipose tissue activation. Temperature. 2018 2(2):147-161. doi: 10.1080/23328940.2017.1397085

25) Robinson L, Ojha S, Symonds ME, Budge H. Body mass index as a determinant of brown adipose tissue function in healthy children. J Pediatr. 2014;164:318-322. doi: 10.1016/j.jpeds.2013.10.005

26) Symonds ME, Henderson K, Elvidge L. Thermal imaging to assess age related changes of skin temperature within the supraclavicular region co-locating with brown adipose tissue in healthy children. J Pediatr. 2012;161:892-898. doi: 10.1016/j.jpeds.2012.04.056

27) Salem V, Izzi-Engbeaya C, Coello C, Thomas DB, Chambers ES, Comninos AN, Buckley A, Win Z, Al-Nahhas A, Rabiner EA, Gunn RN, Budge H, Symonds ME, Bloom SR, Tan TM, Dhillo WS. Glucagon increases energy expenditure independently of brown adipose tissue activation in humans. Diabetes Obes Metab. 2016;18(1):72-81. doi: 10.1111/dom.12585

28) Lee $P$, Ho KK, Lee P, Greenfield JR, Ho KK, Greenfield JR. Hot fat in a cool man: infrared thermography and brown adipose tissue. Diabetes Obes Metab. 2011;13(1):92-93. doi: 10.1111/j.1463-1326.2010.01318.x.

29) Nirengi $S$, Wakabayashi $H$, Matsushita $M$, Domichi M, Suzuki S, Sukino $S$, Suganuma $A$, Kawaguchi Y, Hashimoto T, Saito M, Sakane N. An optimal condition for the evaluation of human brown adipose tissue by infrared thermography. PLoS One. 2019;14(8):e0220574. doi: 10.1371/journal.pone.0220574. eCollection 2019 
30) Gagner M, Hutchinson C, Rosenthal R. Fifth International Consensus Conference: current status of sleeve gastrectomy. Surg Obes Relat Dis. 2016;12(4):750-756. doi: 10.1016/j.soard.2016.01.022

31) Kaijser MA, van Ramshorst GH, Emous $M$, Veeger N, van Wagensveld BA, Pierie JEN. A Delphi Consensus of the Crucial Steps in Gastric Bypass and Sleeve Gastrectomy Procedures in the Netherlands. Obes Surg. 2018;28(9):2634-2643. doi: 10.1007/s11695018-3219-7

32) Sabench Pereferrer F, Dominguez-Adame Lanuza E, Ibarzabal A, Socas Macias M, Valenti Azcarate V, Garcia Ruiz de Gordejuela A, Garcia-Moreno Nisa F, Gonzalez Fernandez J, Vilallonga Puy R, Vilarrasa Garcia N, Sanchez Santos R, Sociedad Espanola de Cirugia de la O. Quality criteria in bariatric surgery: Consensus review and recommendations of the Spanish Association of Surgeons and the Spanish Society of Bariatric Surgery. Cir Esp. 2017;95(1):4-16. doi: 10.1016/j.ciresp.2016.09.007

33) Schauer PR, Bhatt DL, Kirwan JP, Wolski K, Aminian A, Brethauer SA, Navaneethan SD, Singh RP, Pothier CE, Nissen SE, Kashyap SR, Investigators S. Bariatric Surgery versus Intensive Medical Therapy for Diabetes - 5-Year Outcomes. N Engl J Med. 2017;376(7):641-651. doi: 10.1056/NEJMoa1600869

34) Cannon B, Nedergaard J. Brown adipose tissue: function and physiological significance. Physiol Rev. 2004;84(1):277-359. Doi: 10.1152/physrev.00015.2003

35) Sarasniemi JT, Koskensalo K, Raiko J, Nuutila P, Saunavaara J, Parkkola R, Virtanen KA. Skin temperature may not yield human brown adipose tissue activity in diverse populations. Acta Physiol. 2018;224(3):e13095. doi: 10.1111/apha.13095 
Figures 1. Representative IRT images of 2 patients with severe obesity undergoing RYGB or LSG, respectively.

IRT before RYGB at 0 minutes (A) and before RYGB at 5 minutes of cold exposure (B). IRT 6 months after RYGB at 0 minutes (C)and 6 months after RYGB at 5 minutes of cold exposure (D). IRT before LSG at 0 minutes (E) and before LSG at 5 minutes of cold exposure (F). IRT 6 months after LSG at 0 minutes (G) and 6 months after LSG at 5 minutes of cold exposure (H).

Figure 2. BAT thermogenic capacity of patients with severe obesity is increased after BS.

Supraclavicular skin temperature corrected by sternum temperature before and 5 minutes after cold exposure. Thermogenic activation, measured as the difference between 5 minutes and 0 minutes, is represented at basal time and 6 months after the RYGB (A) and SLG (B). Paired twotailed t-test was used. ${ }^{* *} p<0.01$

Supplementary Figure S1. Supraclavicular skin temperature measured in several times during 5 minutes of cold stimulus. Supraclavicular skin temperature (으) before and $1,2,3,4$, and 5 minutes after cold stimulus ( $n=8$ patients). 
Table 1. Anthropometric and metabolic parameters from patients with morbid obesity before and after bariatric surgery.

\begin{tabular}{|c|c|c|c|c|c|c|}
\hline \multirow[b]{2}{*}{1} & \multicolumn{2}{|c|}{ Basal } & \multicolumn{2}{|c|}{6 months } & \multicolumn{2}{|c|}{$\Delta 6-0$ months } \\
\hline & LSG $(n=15)$ & RYGB (n=16) & LSG & RYGB & LSG & RYGB \\
\hline Age (years) & $46 \pm 13$ & $53 \pm 8$ & - & - & - & - \\
\hline $\operatorname{Sex}(F / M)$ & $10 / 5$ & $9 / 7$ & - & - & - & - \\
\hline Type 2 diabetes & 5 & 9 & 0 & 0 & - & - \\
\hline Body mass index $\left(\mathrm{kg} / \mathrm{m}^{2}\right)$ & $46 \pm 9.7$ & $42 \pm 5.1$ & $35 \pm 9.0^{\#}$ & $31 \pm 5.3^{\#}$ & $-11.74 \pm 2.82$ & $-10.91 \pm 1.68$ \\
\hline Waist circumference $(\mathrm{cm})$ & $136 \pm 14$ & $130 \pm 11$ & $117 \pm 16^{\#}$ & $105 \pm 15^{\#}$ & $-19.25 \pm 11.23$ & $-25.96 \pm 11.91$ \\
\hline Glucose (mg/dl) & $94 \pm 10$ & $128 \pm 44 *$ & $86 \pm 9$ & $90 \pm 16^{\#}$ & $-7.60 \pm 9.91$ & $-36.33 \pm 30.68^{*}$ \\
\hline Glycated hemoglobin (\%) & $5.6 \pm 0.38$ & $6.2 \pm 1.01 *$ & $5.3 \pm 0.32$ & $5.3 \pm 0.50^{\#}$ & $-0.30 \pm 0.27$ & $-0.89 \pm 0.61 *$ \\
\hline Insulin (m.u.int./I) & $11.02 \pm 8.48$ & $12.07 \pm 7.32$ & $10.38 \pm 8.34$ & $7.14 \pm 4.27^{\#}$ & $-2.38 \pm 9.32$ & $-10.72 \pm 17.98$ \\
\hline $\begin{array}{l}\text { Homeostatic model assessment } \\
\text { (HOMA-IR) }\end{array}$ & $2.87 \pm 2.21$ & $4.50 \pm 3.99$ & $2.54 \pm 2.11$ & $1.63 \pm 1.13^{\#}$ & $-0.77 \pm 2.3$ & $-4.48 \pm 6.68$ \\
\hline Triacylglycerides (mg/dl) & $133 \pm 46$ & $130 \pm 35$ & $97 \pm 39^{\#}$ & $100 \pm 33^{\#}$ & $-38.50 \pm 54.37$ & $-29.81 \pm 46.28$ \\
\hline Total cholesterol (mg/dl) & $153 \pm 20$ & $145 \pm 33$ & $181 \pm 45$ & $164 \pm 34$ & $30.00 \pm 54.17$ & $18.75 \pm 51.36$ \\
\hline LDL -cholesterol (mg/dl) & $85 \pm 20$ & $82 \pm 34$ & $113 \pm 43$ & $100 \pm 30$ & $30.50 \pm 52.76$ & $15.06 \pm 46.65$ \\
\hline HDL-cholesterol (mg/dl) & $41 \pm 9$ & $37 \pm 4$ & $48 \pm 7$ & $48 \pm 22$ & $8.41 \pm 7.82$ & $10.97 \pm 22.97$ \\
\hline Creatinine $(\mathrm{mg} / \mathrm{dl})$ & $0.91 \pm 0.64$ & $0.84 \pm 0.26$ & $0.86 \pm 0.59$ & $0.76 \pm 0.18$ & $0 \pm 0.15$ & $-0.10 \pm 0.14$ \\
\hline Urea $(\mathrm{mg} / \mathrm{dl})$ & $39.13 \pm 29.09$ & $37.06 \pm 13.74$ & $34.80 \pm 24.27$ & $35.00 \pm 7.65$ & $-4 \pm 9.87$ & $-2.06 \pm 11.62$ \\
\hline C-reactive protein $(\mathrm{mg} / \mathrm{l})$ & $8.89 \pm 5.08$ & $6.67 \pm 5.17$ & $7.12 \pm 7.32$ & $4.22 \pm 3.59$ & $-3 \pm 9.79$ & $0.11 \pm 10.92$ \\
\hline Systolic blood pressure (mmHg) & $136 \pm 16$ & $134 \pm 13$ & - & - & - & - \\
\hline $\begin{array}{l}\text { Diastolic blood pressure } \\
(\mathrm{mmHg})\end{array}$ & $80 \pm 7$ & $80 \pm 10$ & - & - & - & - \\
\hline $\begin{array}{l}\text { Absolute temperature } \\
\text { difference }(\stackrel{\circ}{ } \mathrm{C})^{\text {a }}\end{array}$ & $0.59 \pm 0.2$ & $0.56 \pm 0.2$ & $0.44 \pm 0.1$ & $0.58 \pm 0.3$ & - & - \\
\hline $\begin{array}{l}\text { Relative temperature } \\
\text { difference }(\mathrm{o} C)^{\mathrm{b}}\end{array}$ & $0.06 \pm 0.1$ & $0.09 \pm 0.1$ & $0.32 \pm 0.1^{\#^{*}}$ & $0.08 \pm 0.1$ & - & - \\
\hline
\end{tabular}

Data are shown as mean \pm SD except when it is indicated. Two-tail Student's t-test was applied to compare two groups, and one-way ANOVA with Tukey's post-hoc test was used for comparisons between more than two groups. * indicates $p<0.05$ between LSG and RYGB; \# indicates $p<0.05$ between 0-6 postsurgery. LSG: laparoscopic sleeve gastrectomy; RYGB: roux-en-Y gastric bypass; LDL-Cho: Low-density lipoprotein cholesterol; HDL-Cho: High-density lipoprotein cholesterol.

a This parameter is the difference between the supraclavicular skin temperature measured before the cold stimulation and the supraclavicular skin temperature measured after 5 minutes of cold exposure.

${ }^{b}$ This parameter is the difference between the supraclavicular skin temperature minus the sternum temperature measured before the cold stimulation and the supraclavicular skin temperature minus the sternum temperature measured after 5 minutes of cold exposure. 
Figure 1

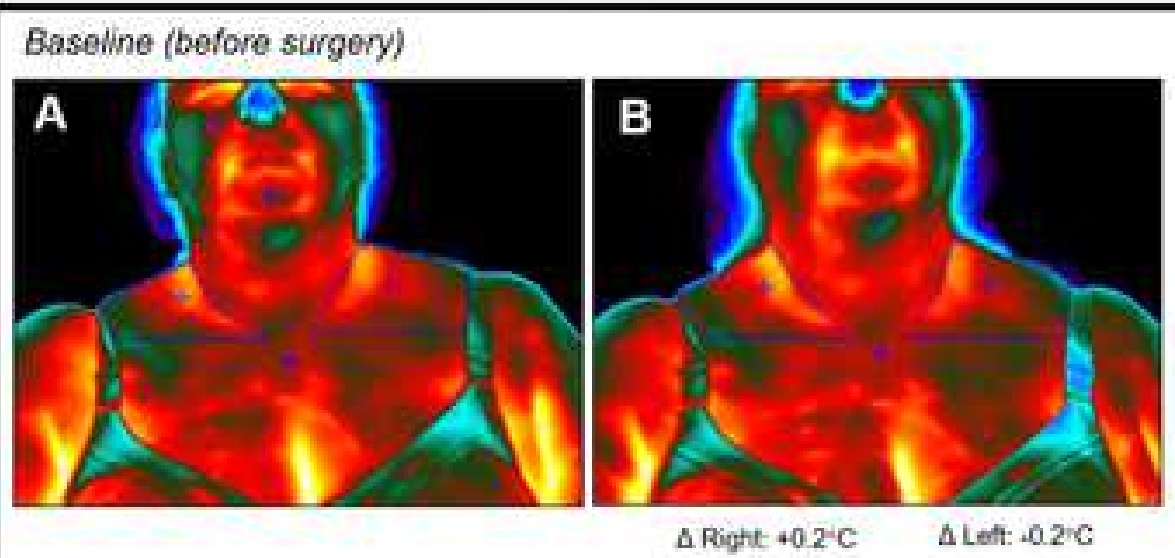

6 months later (affer surgery-Roux-en-Y gastric bypass)

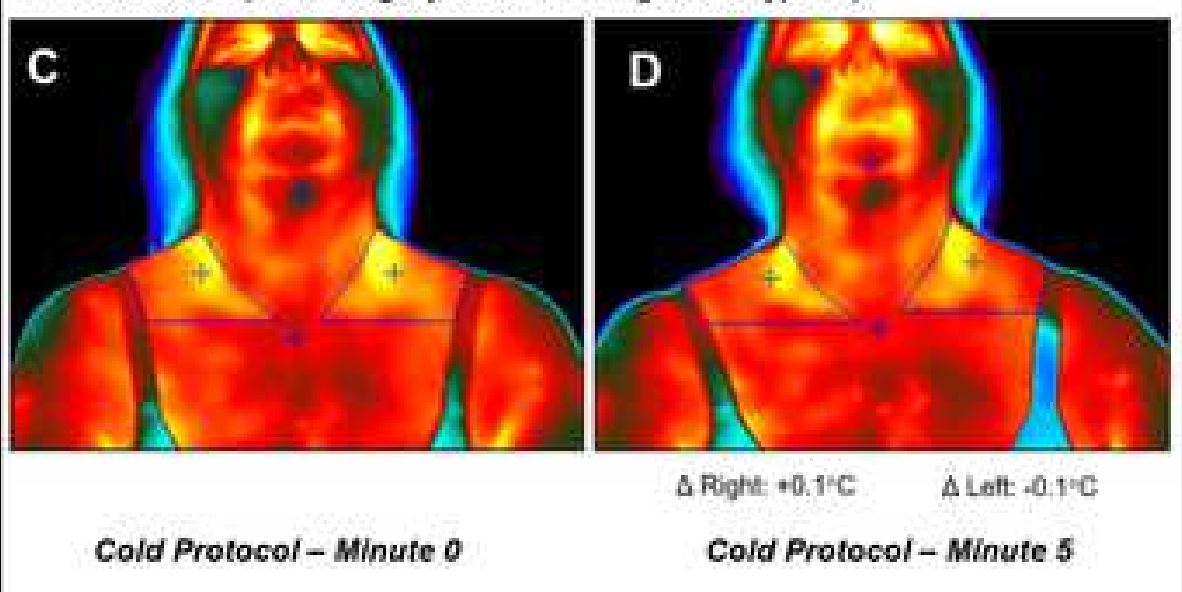

LSG

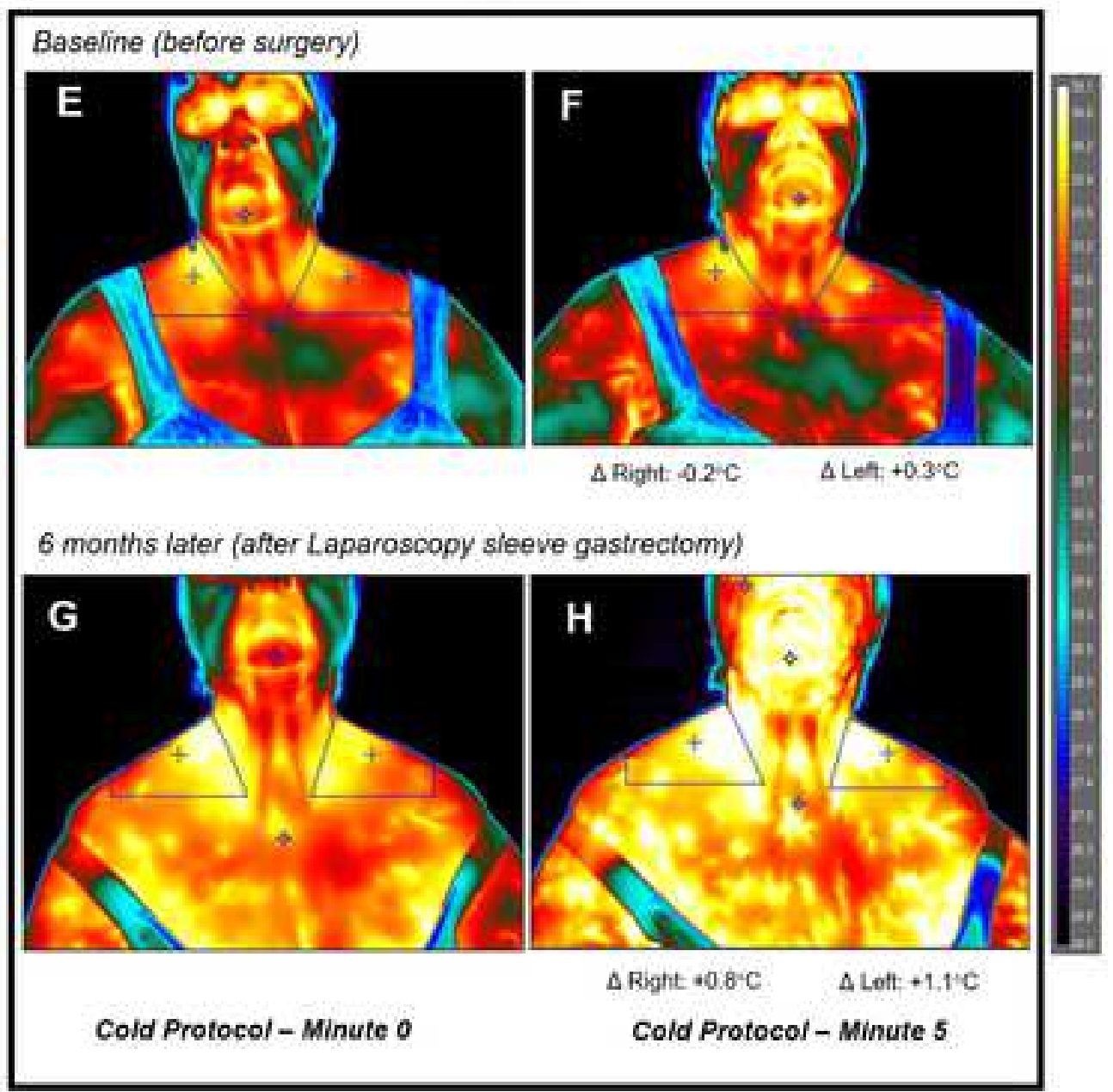




\section{Figure 2}

A)

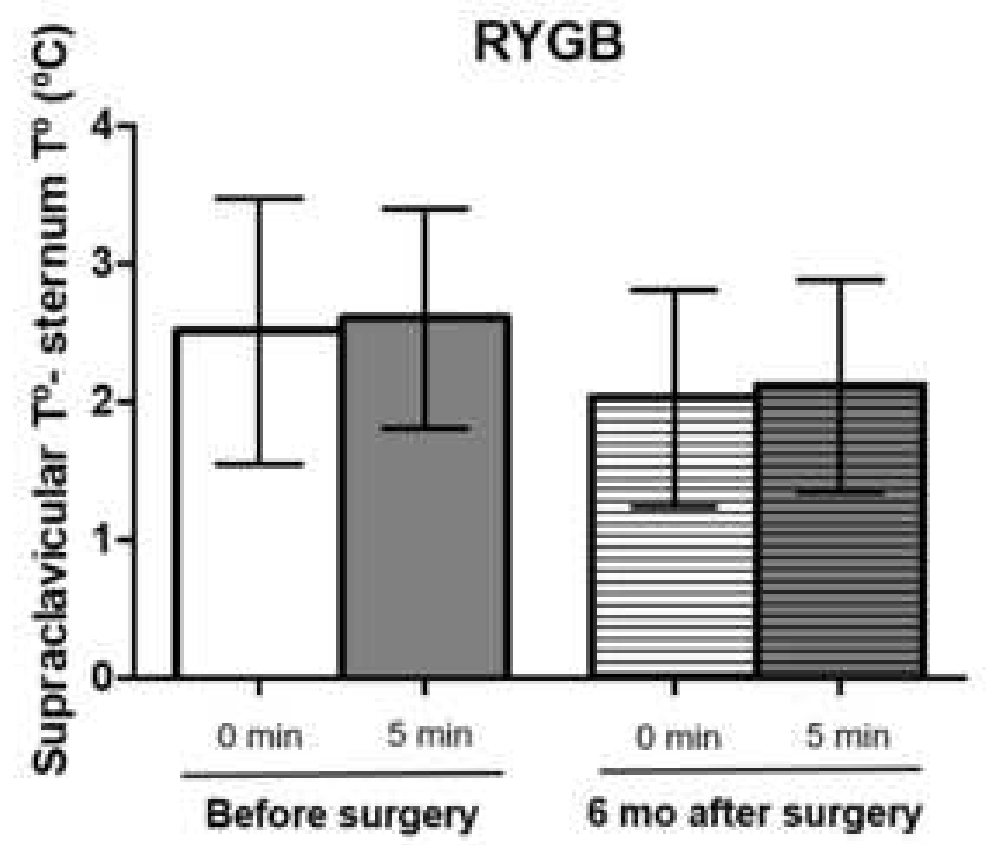

B)

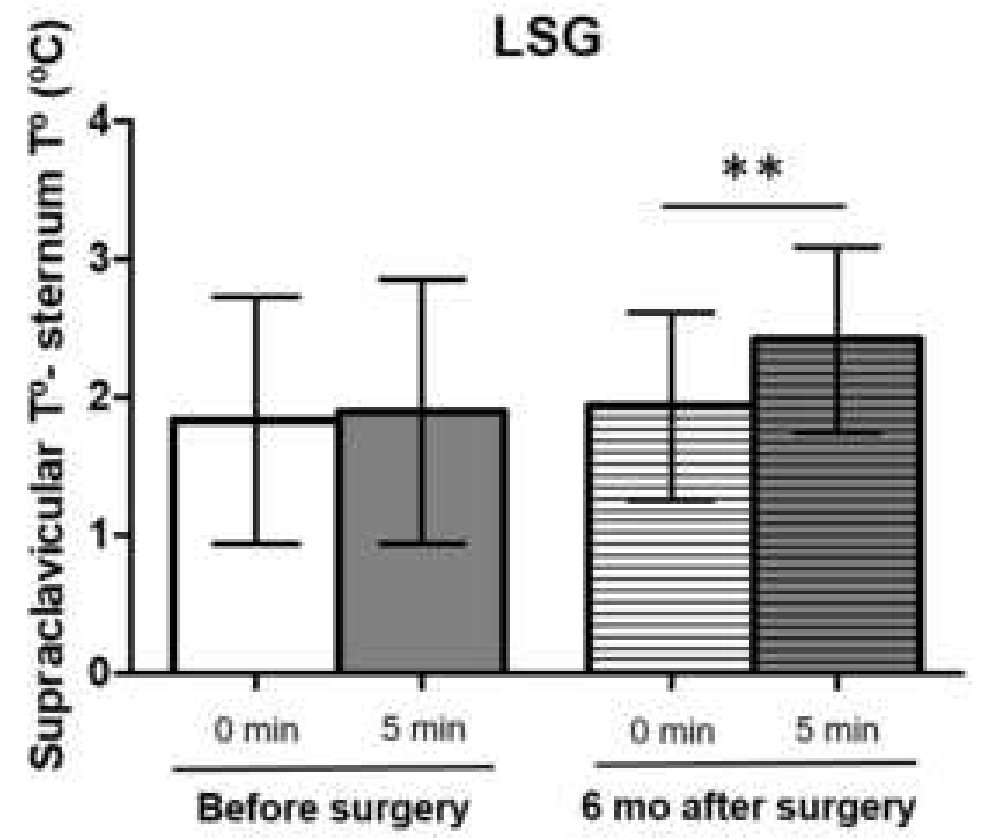




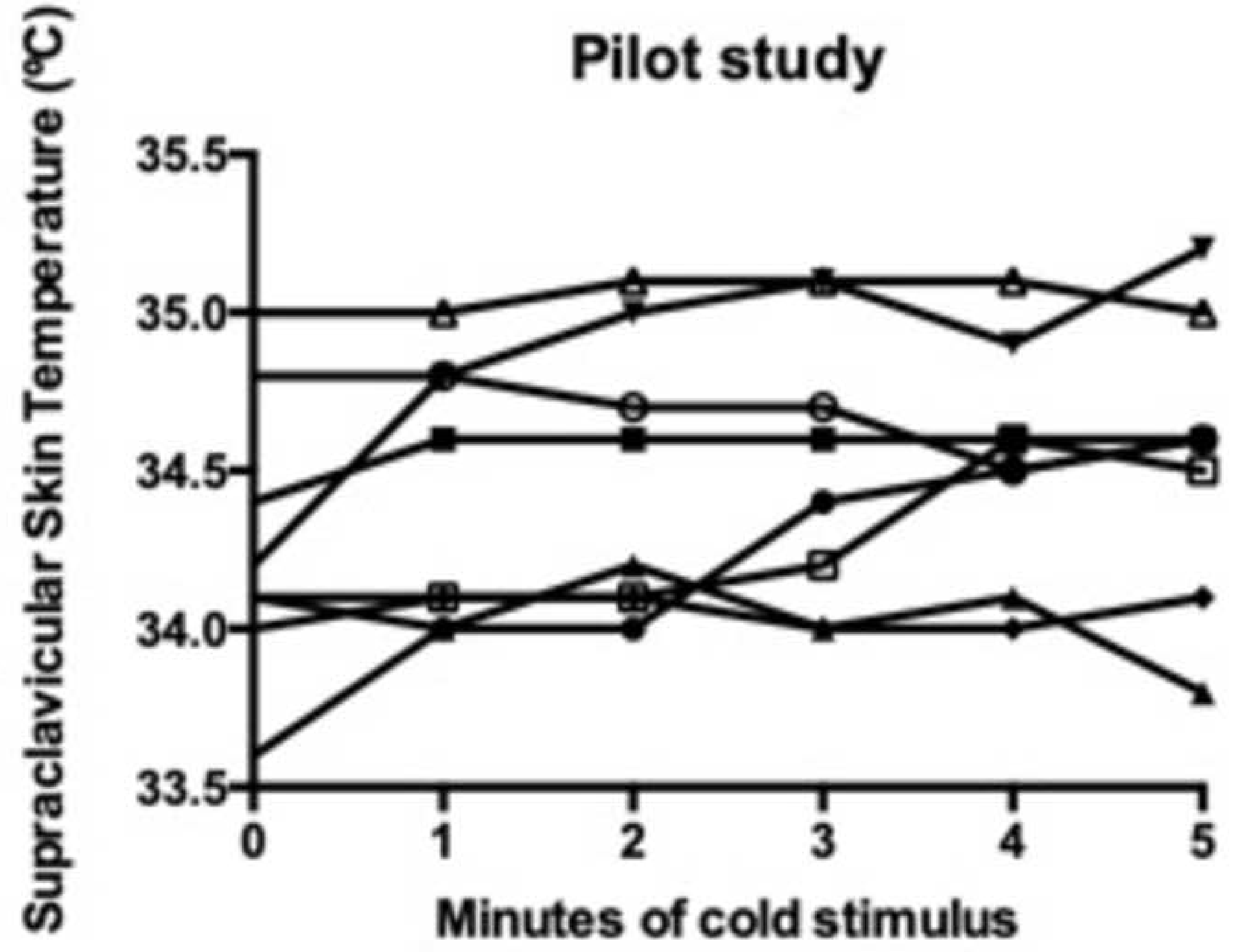

